TRANSACTIONS OF THE

AMERICAN MATHEMATICAL SOCIETY

Volume 350, Number 7, July 1998, Pages 2697-2717

S 0002-9947(98)02108-4

\title{
PERIODIC ORBITS IN MAGNETIC FIELDS AND RICCI CURVATURE OF LAGRANGIAN SYSTEMS
}

\author{
ABBAS BAHRI AND ISKANDER A. TAIMANOV
}

\begin{abstract}
A Lagrangian system describing a motion of a charged particle on a Riemannian manifold is studied. For this flow an analog of a Ricci curvature is introduced, and for Ricci positively curved flows the existence of periodic orbits is proved.
\end{abstract}

\section{INTRODUCTION}

Let $M^{n}$ be a differentiable $n$-dimensional closed manifold endowed with a Riemannian metric $g_{i j}$ and with an exact 2-form $F$.

We will consider the problem of existence of closed extremals of the functional

$$
S(\gamma)=\int_{\gamma}\left(\sqrt{g_{i j} \dot{x}^{i} \dot{x}^{j}}+A_{i} \dot{x}^{i}\right) d t
$$

where $d A=F$, on the space of closed curves on the manifold $M^{n}$. Here $A$ is a 1 -form (i.e., $F$ is an exact 2 -form).

This functional is a natural generalization of the usual functional of length, and its closed extremals correspond to periodic trajectories of the motion of particles on the Riemannian manifold $M^{n}$ when the kinetic energy is defined by the metric tensor and the form $F$ defines a magnetic field. Also this functional corresponds to the periodic orbits for other problems of classical mechanics and mathematical physics, as it was shown in [N2], [N3], [NS].

When the Lagrangian function

$$
\sqrt{g_{i j} \dot{x}^{i} \dot{x}^{j}}+A_{i} \dot{x}^{i}
$$

is everywhere positive we obtain a Finsler metric, and the periodic problem can be studied by the methods of classical Morse theory. This case is very well understood. But otherwise we cannot use these methods, because this functional is not bounded from below and also does not satisfy the Palais-Smale conditions or their analogues.

Its investigation was began by Novikov ([N1], [N2], [N3]) who, in particular, introduced a method of throwing out cycles for overcoming topological difficulties. He also considered the case when the functional $S$ is multi-valued. This happens if a magnetic field is a closed but not exact 2 -form.

Some results about the existence of non-selfintersecting closed extremals on twodimensional manifolds were obtained in [T2], [T3] for strong magnetic fields. A proof of the first result was sketched in $[\mathrm{NT}]$. Later its generalizations were proved

Received by the editors December 28, 1995.

1991 Mathematics Subject Classification. Primary 58E05, 58E30, 49N66.

(C) 1998 American Mathematical Society 
by using other methods in [T2], [T3]. The methods of these papers make strong use of special properties of the two-dimensional case.

Arnold ([A]) and Kozlov ([Ko]) suggested using for this problem methods of symplectic topology (the generalization of the Poincaré theorem which was proposed by Arnold and was proved by Conley and Zehnder ([CZ])). They carried out their program for a positive magnetic field on the flat two-dimensional torus. Notice that Grinevich and Novikov proved that the principle of throwing cycles holds in this situation ([GN]).

Symplectic methods were developed by Ginzburg, who obtained analogous estimates for very strong positive magnetic fields on arbitrary orientable two-dimensional manifolds ([G1], [G2]).

Notice that these results relate to the case then the functional $S$ is multi-valued, and their proofs also used specific properties of the two-dimensional case.

As Ginzburg also noticed, there are no periodic trajectories on two-dimensional Riemann surfaces with genus $\geq 2$ and constant negative curvature, in the magnetic field which is given by an area form on this manifold ([G2]). M. Ratner later pointed out that the paper of Hedlund $([\mathrm{H}])$ contains a complete description of such flows on the Lobachevski upper half plane, for which the magnetic field is proportional to an area form.

We also notice that in the case of exact magnetic fields negative results are not known.

In this paper we suggest another approach to this problem and prove the main theorem which will be formulated below. We give a method of proving existence of periodic orbits on manifolds of arbitrary dimension. This method is based on approximation of the functional $S$ by auxiliary functionals which satisfy the PalaisSmale conditions, and on getting closed extremals as limits of sequences of closed extremals of auxiliary functionals. This works under an additional condition, i.e., in the case when the "Ricci curvature" of this Lagrangian system is positive. One can see that this approach uses ideas of the seminal paper of Sacks and Uhlenbeck ([SU]).

Similar phenomena have been studied in the framework of contact forms, when one has to extremize on Legendre curves for a form $\beta$, the $L^{2}$-norm of $\alpha(x)$, where $\alpha$ is another contact form (see $[\mathrm{B}]$ ).

But first let us introduce some definitions.

Let $R_{j k l}^{i}$ be a Riemann curvature tensor and $\left\{e_{1}, \ldots, e_{n}\right\}$ be an orthonormal basis in a tangent space $T_{x} M^{n}$. Then a Ricci curvature tensor is defined by the formula

$$
K(u, v)=-\sum_{\alpha=1}^{n}\left(R\left(u, e_{\alpha}\right) v, e_{\alpha}\right) .
$$

Now we introduce in the same manner the tensor

$$
H(w)=\sum_{\alpha=1}^{n} G\left(e_{\alpha}, e_{\alpha}, w\right),
$$

where

$$
G(u, v, w)=\left(\nabla_{u} F\right)_{i j} v^{j} w^{k}
$$

Those definitions, as it can be proved, do not depend on the choice of the orthonormal bases $\left\{e_{j}\right\}$ in tangent spaces. 
Main Theorem. Let $M^{n}$ be a closed Riemannian manifold endowed with a Riemannian metric $g_{i j}$ and an exact 2-form $F$ which satisfy the following condition:

$$
\min _{|v|=1}\left\{f(v)=\frac{K(v, v)}{|v|}-H(v)\right\}>0 .
$$

Then there exists a closed non-one-point extremal of the functional

$$
S(\gamma)=\int_{\gamma}\left(\sqrt{g_{i j} \dot{x}^{i} \dot{x}^{j}}+A_{i} \dot{x}^{i}\right) d t
$$

where $d A=F$.

This theorem will be proved in $\S 3$.

Indeed, the method introduced in the present paper gives more: one almost can derive the Morse inequalities for everywhere positive functionals and get the MorseNovikov inequalities for a functional which is somewhere negative (see Theorem 1). This needs some additional work.

We mention that the function $f(v)$ for the case of such Lagrangian systems is the analogue of the Ricci curvature tensor. So, if one can treat a Ricci curvature as an indefinite Riemannian metric tensor, a Ricci curvature of general homogeneous Lagrangian system ought to be treated as an indefinite Finsler metric.

\section{Functional spaCES, AUXiliary FunCtionals, AND THEIR ANALYTIC PROPERTIES}

Let $M^{n}$ be a differentiable $n$-dimensional closed Riemannian manifold. We denote by $g_{i j}$ a metric tensor on $M^{n}$, and by $F$ a closed 2-form on $M^{n}$.

Assume that this form is an exact form, i.e. there exists a 1 -form $A_{i} d x^{i}$ such that $d A=F$ :

$$
F_{i j}=\frac{\partial A_{j}}{\partial x^{i}}-\frac{\partial A_{i}}{\partial x^{j}}
$$

We denote by $\nabla$ the Levi-Civita derivation, i.e. the covariant derivation which is compatible with the metric and has vanishing torsion. The scalar product of tangent vectors $\eta_{1}, \eta_{2} \in T_{x} M^{n}$ we denote by $\left(\eta_{1}, \eta_{2}\right)$.

Let us denote by $H\left(S^{1}, M^{n}\right)$ a Hilbert manifold which is formed by maps $\gamma$ : $[0,1] \rightarrow M^{n}$ such that $\gamma(0)=\gamma(1)$. The mapping $\gamma$ is an $H^{1}$-mapping ([K1], [K2]). The tangent space $T_{\gamma} H\left(S^{1}, M^{n}\right)$ of $H\left(S^{1}, M^{n}\right)$ at $\gamma$ can be identified with the space of $H^{1}$-vector fields $\xi(t)$ along the contour $\gamma$.

We denote by $|\dot{\gamma}|$ a norm of the tangent vector $d \gamma / d t$, and by $\frac{D}{\partial t}$ the covariant derivative along the contour $\gamma$. Now we can introduce the Riemann metric $(\xi, \eta)_{H}$ on fibers of the tangent bundle $T_{\gamma} H\left(S^{1}, M^{n}\right)$ :

$$
(\xi, \eta)_{H}=\int_{0}^{1}\left\{(\xi(t), \eta(t))+\left(\frac{D \xi}{\partial t}(t), \frac{D \eta}{\partial t}(t)\right)\right\} d t
$$

where $\xi, \eta \in T_{\gamma} H\left(S^{1}, M^{n}\right)$.

We see that the group $G$ of orientation-preserved $H^{1}$-homeomorphisms of the circle $S^{1}$ acts naturally on $H\left(S^{1}, M^{n}\right)$. Let us denote by $G_{0}$ its subgroup which is formed by orientation-preserved $H^{1}$-homeomorphisms of the interval $[0,1]$. The factor-spaces $H\left(S^{1}, M^{n}\right) / G_{0}$ and $H\left(S^{1}, M^{n}\right) / G$ we denote by $\Omega\left(M^{n}\right)$ and $P\left(M^{n}\right)$ respectively. They can be identified with spaces of oriented non-parametrized $H^{1}$ contours with based point and unbased point respectively. 
We denote by $H_{0}\left(S^{1}, M^{n}\right), \Omega_{0}\left(M^{n}\right)$, and $P_{0}\left(M^{n}\right)$ the components of these spaces formed by the contours homotopic to zero.

The scheme of the proof of the main results is as follows. First we will introduce the auxiliary functionals $S_{\varepsilon, \tau}$ which become the functional

$$
S(\gamma)=\int_{\gamma}\left(|\dot{\gamma}|+A_{i} \dot{\gamma}^{i}\right) d t
$$

when $\varepsilon, \tau \rightarrow 0$. These functionals will satisfy the Palais-Smale conditions. After that we will show that, for sufficiently small positive values of $\varepsilon$ and $\tau$, to every nontrivial cycle $H_{*}\left(H_{0}\left(S^{1}, M^{n}\right),\{S \leq 0\}\right)$ there corresponds a critical point of bounded Morse index of the functional $S_{\varepsilon, \tau}$. We will find conditions, on metrics and 2-forms $F$, which will imply

$$
\text { length }(\gamma)<\text { const index }(\gamma)
$$

for critical points of the functionals $S_{\varepsilon, \tau}$ for sufficiently small values of $\varepsilon$ and $\tau$. Under these conditions we will prove that there exists a sequence of critical points $\gamma_{\varepsilon_{k}, \tau_{k}}$, of the functionals $S_{\varepsilon_{k}, \tau_{k}}$, such that their indices and the lengths of these extremals are uniformly bounded from above, $\varepsilon_{k}, \tau_{k} \rightarrow 0$ as $k \rightarrow \infty$. In the space of contours on $M^{n}$ these extremals tend to closed non-trivial extremals of the functional $S$.

Let introduce now the main functionals:

$$
\begin{gathered}
\Phi(\gamma)=\int_{0}^{1} A_{j} \dot{\gamma}^{j} d t, \\
E_{\theta}: H\left(S^{1}, M^{n}\right) \rightarrow \mathbf{R}, \quad 0 \leq \theta \leq 1,
\end{gathered}
$$

where

$$
E_{\theta}(\gamma)=\int_{0}^{1}|\dot{\gamma}|^{1+\theta} d t
$$

$E_{0}$ is the usual functional of length which is $G$-invariant.

The main auxiliary functionals which we will consider are the following functionals:

$$
S_{\varepsilon, \tau}=\varepsilon E_{1}+E_{\tau}+\Phi
$$

where $\varepsilon>0,0<\tau<1$.

Given a functional $D \in\left\{E_{\theta}, \Phi\right\}$, we denote by $\delta D(\gamma)$ and $\delta^{2} D(\gamma)$ its first and second variational derivatives at $\gamma$, respectively.

The method of computing of these derivatives is very useful ([K1], [K2], [M]). Let us denote by exp :TM $\rightarrow M^{n}$ the exponential mapping : $(x, v) \rightarrow \exp (x, v) \in$ $M^{n}, x \in M^{n}, v \in T_{x} M^{n}$, which corresponds to the Riemannian metric $g_{i j}$ on $M^{n}$. By $V_{\gamma, \xi, \eta}(r, s)$ we denote the following two-dimensional family of contours:

1) $V_{\gamma, \xi, \eta}(r, s):[0,1] \rightarrow M^{n}, V_{\gamma, \xi, \eta}(r, s)(0)=V_{\gamma, \xi, \eta}(r, s)(1)$;

2) $V_{\gamma, \xi, \eta}(r, s)(t)=\exp (\gamma(t), r \xi(t)+s \eta(t))$, where $\xi, \eta \in T_{\gamma} H\left(S^{1}, M^{n}\right), r, s \in \mathbf{R}$.

Since any closed curve which is sufficiently close to $\gamma$ is represented in the form $V_{\gamma, \xi, 0}(1,0)(t)$ and since the exponential mapping determines local charts in the Hilbert manifold $H\left(S^{1}, M^{n}\right)$, we have the following lemma. 
Lemma 1.

$$
\begin{gathered}
\delta S(\gamma)(\xi)=\left.\left(\partial S\left(V_{\gamma, \xi, 0}(r, 0)\right) / \partial r\right)\right|_{r=0}, \\
\delta^{2} S(\gamma)(\xi, \eta)=\left.\left(\partial^{2} S\left(V_{\gamma, \xi, \eta}(r, s)\right) / \partial r \partial s\right)\right|_{r=s=0} .
\end{gathered}
$$

Now we are able to compute variational derivatives of the functionals $S_{\varepsilon, \tau}$.

Lemma 2. Let $\theta>0$. Then $E_{\theta} \in C^{1}\left(H\left(S^{1}, M^{n}\right), \mathbf{R}\right)$ and

$$
\delta E_{\theta}(\gamma)(\xi)=(1+\theta) \int_{0}^{1}\left(\frac{D \xi}{\partial t}, \dot{\gamma}\right)|\dot{\gamma}|^{\theta-1} d t .
$$

If also $\gamma \in C^{2}\left(S^{1}, M^{n}\right)$ and the vector-field $\xi(t)$ is of class $C^{1}$, then the formula

$$
\delta E_{\theta}(\gamma)(\xi)=-(1+\theta)\left\{\int_{0}^{1}\left(\xi, \frac{D \dot{\gamma}}{\partial t}\right)|\dot{\gamma}|^{\theta-1} d t+(\theta-1) \int_{0}^{1}(\xi, \dot{\gamma})\left(\frac{D \dot{\gamma}}{\partial t}, \dot{\gamma}\right)|\dot{\gamma}|^{\theta-3} d t\right\}
$$

holds.

Proof of Lemma 2. For the sake of simplicity, let us denote $V_{\gamma, \xi, 0}$ by $V_{\gamma}$. $0)$,

Since the covariant derivation is compatible with the Riemannian metric $\left(\nabla g_{i j} \equiv\right.$

$$
\frac{D}{\partial t}(\alpha, \beta)=\left(\frac{D \alpha}{\partial t}, \beta\right)+\left(\alpha, \frac{D \beta}{\partial t}\right)
$$

for every pair of $H^{1}$-vector-fields $\alpha$ and $\beta$ along $\gamma$. In the sequel we will use this formula for vector-fields along curves of the form $r \rightarrow \exp (\gamma(t), r \xi(t))$. Since $V_{\gamma, \xi, 0}$ can be considered to be a surface parametrized by $t$ and $r$, the Lie bracket $\left[\frac{\partial}{\partial t}, \xi\right]$ is zero for a suitable parametrization. Therefore, the covariant derivation has vanishing torsion, and we derive

$$
\nabla_{\xi} \frac{\partial V_{\gamma}}{\partial t}=\frac{D}{\partial r} \frac{\partial V_{\gamma}}{\partial t}=\frac{D \xi}{\partial t}
$$

Now by using Lemma 1 we obtain

$$
\begin{aligned}
\delta E_{\theta}(\gamma)(\xi) & =\left.\frac{\partial}{\partial r}\left(\int_{0}^{1}\left(\dot{V}_{\gamma}, \dot{V}_{\gamma}\right)^{(\theta+1) / 2} d t\right)\right|_{r=0} \\
= & (1+\theta) \int_{0}^{1}\left(\left.\left(\nabla_{\xi} \dot{V}_{\gamma}, \dot{\gamma}\right)\right|_{r=0}\right)|\dot{\gamma}|^{\theta-1} d t=(1+\theta) \int_{0}^{1}\left(\frac{D \xi}{\partial t}, \dot{\gamma}\right)|\dot{\gamma}|^{\theta-1} d t,
\end{aligned}
$$

where we denote by $\dot{V}_{\gamma}$ the covariant derivative $\frac{\partial V_{\gamma}}{\partial t}$.

$\gamma \in C^{2}\left(S^{1}, M^{n}\right)$ and the vector-field $\xi$ is of class $C^{1}$. We integrate by parts, and derive

$$
\begin{aligned}
& (1+\theta) \int_{0}^{1}\left(\frac{D \xi}{\partial t}, \dot{\gamma}\right)|\dot{\gamma}|^{\theta-1} d t(1+\theta) \int_{0}^{1}\left(\frac{D}{\partial t}(\xi, \dot{\gamma})-\left(\xi, \frac{D}{\partial t} \dot{\gamma}\right)\right)|\dot{\gamma}|^{\theta-1} d t \\
& =-(1+\theta) \int_{0}^{1}\left(\xi, \frac{D}{\partial t} \dot{\gamma}\right)|\dot{\gamma}|^{\theta-1} d t-(1+\theta) \int_{0}^{1}(\xi, \dot{\gamma})\left(\frac{D}{\partial t}|\dot{\gamma}|^{\theta-1}\right) d t
\end{aligned}
$$

We have

$$
\frac{D}{\partial t}|\dot{\gamma}|^{\theta-1}=(\theta-1)\left(\frac{D}{\partial t} \dot{\gamma}, \dot{\gamma}\right)|\dot{\gamma}|^{\theta-3} .
$$

The claim about the smoothness of $E_{\theta}$ follows from the formula for $\delta E_{\theta}$ immediately. 
Lemma 2 is established.

\section{Lemma 3.}

$$
\delta \Phi(\gamma)(\xi)=\int_{0}^{1} F_{i j} \xi^{i} \dot{\gamma}^{j} d t
$$

Proof of Lemma 3. Let us divide the contour $\gamma$ into $\operatorname{arcs} \gamma_{\alpha}, 1 \leq \alpha \leq k$, such that in a neighborhood of every arc $\gamma_{\alpha}$ it is possible to introduce local coordinates and make sense of expressions of the form $\partial \xi^{j} / \partial t$ which are not covariant. We have

$$
\begin{aligned}
\delta \Phi(\gamma)(\xi)=\left.\frac{d}{d r}\left(\int_{0}^{1} A_{i} \dot{V}_{\gamma}^{i}(r, 0)\right)\right|_{r=0} \\
=\int_{0}^{1}\left(\nabla_{\xi} A_{i}\right) \dot{\gamma}^{i} d t+\left.\int_{0}^{1} A_{i}\left(\nabla_{\xi} \dot{V}_{\gamma}^{i}(r, 0)\right)\right|_{r=0} d t \\
=\sum_{\alpha=1}^{k}\left\{\int_{\gamma_{\alpha}}\left(\partial_{j} A_{i}-\Gamma_{j i}^{k} A_{k}\right) \xi^{j} \dot{\gamma}^{i} d t+\int_{\gamma_{\alpha}} A_{i} \frac{D \xi^{i}}{\partial t} d t\right\} \\
=\sum_{\alpha=1}^{k}\left\{\int_{\gamma_{\alpha}}\left(\partial_{j} A_{i}-\Gamma_{j i}^{k} A_{k}\right) \xi^{j} \dot{\gamma}^{i} d t+\int_{\gamma_{\alpha}} A_{i}\left(\frac{\partial \xi^{i}}{\partial t}+\Gamma_{j k}^{I} \xi^{j} \dot{\gamma}^{k}\right) d t\right\} \\
=\sum_{\alpha=1}^{k} \int_{\gamma_{\alpha}}\left(\partial_{j} A_{i} \xi^{j} \dot{\gamma}^{i}+A_{i} \frac{\partial \xi^{i}}{\partial t}\right) d t
\end{aligned}
$$

where $\Gamma_{j k}^{i}$ are the Christoffel symbols which correspond to the connectedness $\nabla$.

Using integration by parts, we obtain

$$
\delta \Phi(\gamma)(\xi)=\sum_{\gamma \alpha}^{k} \int_{\gamma_{\alpha}}\left(\partial_{i} A_{j}-\partial_{j} A_{i}\right) \xi^{i} \dot{\gamma}^{j} d t=\int_{0}^{1} F_{i j} \xi^{i} \dot{\gamma}^{j} d t
$$

Lemma 3 established.

To compute the Morse indices of the extremals of functionals $S_{\varepsilon, \tau}$ it is sufficient to find the second derivatives of these functionals at the critical points.

We start with the following fact. If $\gamma$ is a critical point of the functional $S_{\varepsilon, \tau}$, then the motion of a point along this contour is determined by the Euler-Lagrange equations with the Lagrange function

$$
L_{\varepsilon, \tau}(x, \dot{x})=\varepsilon|\dot{x}|^{2}+|\dot{x}|^{1+\tau}+A_{i}(x) \dot{x}^{i} .
$$

The following lemma holds.

Lemma 4. If $x(t)$ is a trajectory of a system with the Lagrange function $L_{\varepsilon, \tau}$, where $\varepsilon, \tau>0$, then $|\dot{x}|=$ const.

Proof of Lemma 4. By the Euler-Lagrange equations, the function $I=\dot{x}^{i} \frac{\partial L}{\partial \dot{x}^{i}}-L$ is constant along the trajectories. In our case $I=\varepsilon|\dot{x}|^{2}+\tau|\dot{x}|^{1+\tau}$, and thus we obtain that $|\dot{x}|$ is constant along the trajectories.

Lemma 4 follows.

The following lemma now immediately follows from Lemmas 2, 3, and 4, which give this form for the Euler-Lagrange equation. 
Lemma 5. The extremals of the functional $S_{\varepsilon, \tau}$ satisfy the following equation:

$$
\left(\frac{D \dot{\gamma}}{\partial t}\right)^{j}=\frac{\partial}{\partial t} \dot{\gamma}+\Gamma_{i k}^{j} \dot{\gamma}^{i} \dot{\gamma}^{k}=\frac{g^{j k} F_{k i} \dot{\gamma}^{i}}{2 \varepsilon+(1+\tau)|\dot{\gamma}|^{\tau-1}} .
$$

Let us now recall the definition of the Riemann curvature tensor:

$$
R_{j k l}^{i} \xi^{j} \eta^{k} \zeta^{l}=[R(\xi, \eta) \zeta]^{i}=\left(\nabla_{\xi} \nabla_{\eta} \zeta-\nabla_{\eta} \nabla_{\xi} \zeta-\nabla_{[\xi, \eta]} \zeta\right)^{i},
$$

where $\xi, \eta$ and $\zeta$ are smooth vector-fields on $M^{n}$.

Lemma 6. Let $\gamma$ be a nontrivial critical point of the functional $S_{\epsilon, \tau}$. Then at the point $\gamma \in H\left(S^{1}, M^{n}\right)$ the second variational derivative of the functional $S_{\varepsilon, \tau}$ exists, and

$$
\begin{aligned}
\delta^{2} S_{\varepsilon, \tau}(\gamma)(\xi, \eta)= & 2 \varepsilon \int_{0}^{1}\left(\left(\frac{D \xi}{\partial t}, \frac{D \eta}{\partial t}\right)+(R(\eta, \dot{\gamma}) \xi, \dot{\gamma})\right) d t \\
& +(1+\tau) \int_{0}^{1}\left\{(\tau-1)|\dot{\gamma}|^{\tau-3}\left(\frac{D \xi}{\partial t}, \dot{\gamma}\right)\left(\frac{D \eta}{\partial t}, \dot{\gamma}\right)\right. \\
& \left.+|\dot{\gamma}|^{\tau-1}\left(\left(\frac{D \xi}{\partial t}, \frac{D \eta}{\partial t}\right)+(R(\eta, \dot{\gamma}) \xi, \dot{\gamma})\right)\right\} d t \\
& +\int_{0}^{1}\left\{\left(\nabla_{\eta} F\right)_{j k} \xi^{j} \dot{\gamma}^{k}+F_{j k} \xi^{j}\left(\frac{D \eta}{\partial t}\right)^{k}\right\} d t .
\end{aligned}
$$

Proof of Lemma 6. By Lemmas 1 and 2, we have

$$
\begin{aligned}
\delta^{2} E_{\theta}(\gamma)(\xi, \eta)= & \left.(1+\theta) \int_{0}^{1} \frac{D}{\partial s}\left(\left(\frac{D \xi}{\partial t}, \dot{V}_{\gamma}\right)\left|\dot{V}_{\gamma}\right|^{\theta-1}\right)\right|_{s=r=0} d t \\
= & (1+\theta) \int_{0}^{1}\left\{(\theta-1)|\dot{\gamma}|^{\theta-3}\left(\frac{D}{\partial s} \dot{V}_{\gamma}, \dot{\gamma}\right)\left(\frac{D \xi}{\partial t}, \dot{\gamma}\right)\right. \\
& \left.+|\dot{\gamma}|^{\theta-1}\left(\left(\frac{D}{\partial s} \frac{D}{\partial t} \xi, \dot{\gamma}\right)+\left(\frac{D \xi}{\partial t}, \frac{D}{\partial s} \dot{V}_{\gamma}\right)\right)\right\}\left.d t\right|_{s=r=0} \\
= & (1+\theta) \int_{0}^{1}\left\{(\theta-1)|\dot{\gamma}|^{\theta-3}\left(\frac{D \xi}{\partial t}, \dot{\gamma}\right)\left(\frac{D \eta}{\partial t}, \dot{\gamma}\right)\right. \\
& \left.+|\dot{\gamma}|^{\theta-1}\left(\frac{D}{\partial t} \frac{D}{\partial s} \xi+R(\eta, \dot{\gamma}) \xi, \dot{\gamma}\right)+|\dot{\gamma}|^{\theta-1}\left(\frac{D \xi}{\partial t}, \frac{D \eta}{\partial t}\right)\right\} d t
\end{aligned}
$$

Here we use the following fact: if we introduce the coordinates $s$ and $t$ on a surface immersed into $M^{n}$ (in our case $(s, t) \rightarrow \exp (\gamma(t), s \eta(t))$ ), then, by definition of the Riemannian curvature, we have

$$
\frac{D}{\partial s} \frac{D}{\partial t} \xi-\frac{D}{\partial t} \frac{D}{\partial s} \xi=R(\eta, \dot{\gamma}) \xi .
$$

By Lemmas 1 and 3, we also have

$$
\begin{aligned}
\delta^{2} \Phi(\gamma)(\xi, \eta) & =\left.\int_{0}^{1} \frac{D}{\partial s}\left(F_{j k} \xi^{j}\left(V_{\gamma}\right)^{k}\right)\right|_{s=r=0} d t \\
& =\int_{0}^{1}\left\{\left(\nabla_{\eta} F\right)_{j k} \xi^{j} \dot{\gamma}^{k}+F_{j k}\left(\nabla_{\eta} \xi\right)^{j} \dot{\gamma}^{k}+F_{j k} \xi^{j}\left(\nabla_{\eta} \dot{\gamma}\right)^{k}\right\} d t .
\end{aligned}
$$

But

$$
\nabla_{\eta} \dot{\gamma}=\frac{D}{\partial t} \eta
$$


We obtain the formula for the second derivative of $S_{\varepsilon, \tau}$ by adding the formulas for the second derivatives of $\varepsilon E_{1}, E_{\tau}$ and $\Phi$. We do not give this formula here, but show how it reduces to $(2.2)$.

Let us consider terms in this formula that contain $\nabla_{\eta} \xi=\frac{D}{\partial s} \xi$ :

$$
J=\int_{0}^{1}\left(2 \varepsilon+(1+\tau)|\dot{\gamma}|^{\tau-1}\right)\left(\frac{D}{\partial t} \nabla_{\eta} \xi, \dot{\gamma}\right) d t+\int_{0}^{1} F_{j k}\left(\nabla_{\eta} \xi\right)^{j} \dot{\gamma}^{k} d t .
$$

Integrating the first summand by parts and using (2.1), we derive that $J=0$.

The sum of the other terms gives (2.2).

Lemma 6 follows.

The following lemma is a consequence of Lemmas 4 and 6 .

Lemma 7. Let $\gamma$ be a nontrivial critical point of the functional $S_{\varepsilon, \tau}$. Then there exists a neighborhood of $\gamma$ such that in this neighborhood the second derivative of the functional $S_{\varepsilon, \tau}$ exists and is defined by formula (2.2) on this whole neighborhood.

It is easy to see that the neighborhoods formed by contours which are close to $\gamma$ and which have a never vanishing tangent vector satisfy the requirements of this lemma.

\section{Proof of the Main theorem}

A) Preliminaries. First let us prove the following fact.

Lemma 8. The subset of $H\left(S^{1}, M^{n}\right)$ formed by one-point-contours is a manifold of local minima of the functional $S_{\varepsilon, \tau}, 0 \leq \tau<1$.

Proof of Lemma 8. Using the Hölder inequality, we get

$$
\left(\int_{0}^{1} f(t) d t\right)^{1+\tau} \leq \int_{0}^{1} f(t)^{1+\tau} d t
$$

for $f \in L_{2}[0,1], f \geq 0$. Substituting $|\dot{\gamma}|$ for $f(t)$ and taking into account that by well-known results from the theory of isoperimetric inequalities the inequality

$$
\int_{\Pi} F \leq c \text { length }(\gamma)^{2},
$$

holds for sufficiently small contours $\gamma$, where $\Pi$ is a sufficiently small surface having $\gamma$ as boundary, and $c$ is a positive constant which depends on the metric $g_{i j}$ and the 2 -form $F$, we obtain the inequality

$$
S_{\varepsilon, \tau} \geq \text { length }(\gamma)^{1+\tau}-\text { c length }(\gamma)^{2},
$$

which holds for all sufficiently small contours from $H_{0}\left(S^{1}, M^{n}\right)$ since every such contour can be transformed into a smooth contour by a perturbation as small as we may wish. Lemma 8 follows from the equality (3.2).

Definition. A nontrivial cycle $\alpha \in H_{*}\left(M^{n}\right)$ can be thrown out into the domain $\left\{S_{\varepsilon, \tau}<0\right\}$ if and only if there exists a continuous mapping

$$
\phi: K \times[0,1] \rightarrow H_{0}\left(S^{1}, M^{n}\right)
$$

such that

1) $K$ is a polyhedron and $[\phi(K \times 1)]=\alpha$, i.e. an image of $K \times 0$ realizes a cycle $\alpha$; and

2) $\phi(K \times 0) \subset\left\{S_{\varepsilon, \tau}<0\right\}$. 
The following lemma holds.

Lemma 9. If $\tau<1$ and a cycle $\alpha \in H_{k}\left(M^{n}\right) \backslash\{0\}$ can be thrown out into $\left\{S_{\varepsilon, \tau}<\right.$ $0\}$, then

1) there exists a positive constant $c_{\varepsilon, \tau}(\alpha)$ (the "critical value" of the functional $S_{\varepsilon, \tau}$ ) defined by the formula

$$
c_{\varepsilon, \tau}(\alpha)=\inf _{\phi \in A(\alpha)} \max \left\{S_{\varepsilon, \tau}(\gamma): \gamma \in \operatorname{Im} \phi\right\}>0,
$$

where $A(\alpha)$ is the set of all possible throwings out of a cycle $\alpha$;

2) $H_{k+1}\left(H_{0}\left(S^{1}, M^{n}\right),\left\{S_{\varepsilon, \tau} \leq 0\right\}\right) \neq 0$;

3) if $D_{k}$ is the linear span of $k$-cycles (with coefficients from the field $E$ ) which can be thrown out into $\left\{S_{\varepsilon, \tau}<0\right\}$, then

$$
\operatorname{dim}_{E} H_{k+1}\left(H_{0}\left(S^{1}, M^{n}\right),\left\{S_{\varepsilon, \tau} \leq 0\right\} ; E\right) \geq \operatorname{dim}_{E} D_{k} .
$$

For proof of this lemma see [T1], [T2]. We also will use the results of these papers in the sequel.

Lemma 10. Let there exist a contour $\gamma \in \Omega_{0}\left(M^{n}\right)$ such that $\left(E_{0}+\Phi\right)(\gamma)<0$. Then there exist positive constants $\varepsilon_{0}$ and $\tau_{0}$ such that all the manifold $M^{n}$ is thrown out into $S_{\varepsilon, \tau}<0$ for $\varepsilon<\varepsilon_{0}, \tau<\tau_{0}$.

Proof of Lemma 10. By the construction of the throwing out given in [T1], the contours from $\phi\left(M^{n} \times[0,1]\right)$ are piecewise-smooth and we can assume that they lie in $H_{0}\left(S^{1}, M^{n}\right)$.

Since the subset $\phi\left(M^{n} \times 0\right)$ is the continuous image of the compact space, there exist sufficiently small positive constants $\varepsilon_{0}$ and $\tau_{0}$ such that for $\varepsilon<\varepsilon_{0}, \tau<\tau_{0}$ and for any contour $\gamma \in \phi\left(M^{n} \times 0\right)$ the inequality

$$
\left(\varepsilon E_{1}+E_{\tau}+\Phi\right)(\gamma)<0
$$

holds.

Lemma 10 is proved.

Lemma 11. Let the conditions of Lemma 10 hold. Assume that the manifold $M^{n}$ can be thrown out into $\left\{S_{\varepsilon, \tau}<0\right\}$ for $0<\varepsilon<\varepsilon_{0}$ and $0<\tau, \tau_{0}$. Then we can define a map which corresponds non-zero cycles of $H_{*}\left(M^{n}\right)$ to critical values of the functional $S_{\epsilon, \tau}$ :

$$
\alpha \in H_{*}\left(M^{n}\right) \backslash\{0\} \rightarrow c_{\varepsilon, \tau}(\alpha) .
$$

This mapping is semicontinuous above with respect to the variables $\varepsilon$ and $\tau$.

Proof of Lemma 11. It is sufficient to prove that for any constants $\varepsilon, \tau$ such that $\varepsilon \in\left(0, \varepsilon_{0}\right), \tau \in\left(0, \tau_{0}\right)$ and for any $\omega>0$ there exist positive constants $\lambda$ and $\mu$ such that

$$
c_{\varepsilon^{\prime}, \tau^{\prime}}(\alpha)-c_{\varepsilon, \tau}(\alpha)<\omega
$$

for $\left|\varepsilon-\varepsilon^{\prime}\right|<\lambda$ and $\left|\tau-\tau^{\prime}\right|<\mu$.

By the definitions of the throwing out of cycles and critical values $c_{\varepsilon, \tau}(\alpha)$ corresponding to them, there exists the continuous mapping

$$
\phi: K \times[0,1] \rightarrow H_{0}\left(S^{1}, M^{n}\right),
$$

such that

1) $[\phi(K \times 1]=\alpha$; 
2) $\phi(K \times 1) \subset\left\{S_{\varepsilon, \tau}<0\right\}$;

3) $c_{\varepsilon, \tau} \leq \max \left\{S_{\varepsilon, \tau}(\gamma): \gamma \in \operatorname{Im} \phi\right\} \leq c_{\varepsilon, \tau}+\omega / 2$.

Since the subspaces $\operatorname{Im} \phi$ and $\phi(K \times 0)$ are continuous images of compact spaces, it is evident that such constants $\lambda$ and $\mu$ exist.

Lemma 11 follows.

Lemma 12. Let $\varepsilon$ and $\tau$ be sufficiently small. Then there exists a positive constant $J_{0}$ such that the lengths of extremals of the functionals $S_{\epsilon, \tau}$ which are not points are not less than $J_{0}$.

Proof of Lemma 12. By Lemma 5, extremals of the functional $S_{\varepsilon, \tau}$ satisfy the Euler-Lagrange equation

$$
\frac{\partial}{\partial t} \dot{\gamma}^{j}+\Gamma_{i k}^{j} \dot{\gamma}^{i} \dot{\gamma}^{k}=g^{j k} F_{k i} \dot{\gamma}^{i} /\left(2 \varepsilon+(1+\tau)|\dot{\gamma}|^{\tau-1}\right) .
$$

By Lemma 4, these extremals are naturally parametrized and

$$
\text { length }(\gamma)=|\dot{\gamma}|=\text { const. }
$$

Let $\lambda$ be sufficiently small and let an extremal $\gamma$ lie in a small neighborhood of the contour $\gamma(0)$ endowed with local coordinates $\left(x^{1}, \ldots, x^{n}\right)$ with $\dot{\gamma}=(\lambda, 0, \ldots, 0), \lambda=$ $|\dot{\gamma}|$. By (3.3),

$$
\dot{\gamma}(t)=\dot{\gamma}(0)+o\left(\lambda^{3 / 2}\right)
$$

for $\tau<1 / 2$, and, thus, if $\lambda$ is sufficiently small, then $\dot{\gamma}^{1}$ is nowhere equal to zero and an extremal $\gamma$ cannot be closed.

Lemma 12 follows.

\section{B) Validity of the Palais-Smale conditions for the functionals $S_{\varepsilon, \tau}$.}

(PS) Condition ([PS]). A $C^{1}$-functional $S$ on a Hilbert manifold $X$ satisfies the $(P S)$ condition if and only if for every subset $W \subset X$ on which the functional is bounded from above and the norms of its first derivatives $\|S\|$ are not bounded from below there exists a point $x$ in the closure of $W$ satisfying $\delta S(x)=0$.

First let us mention very simple fact.

Lemma 13. $E_{0}^{2}(\gamma) \leq E_{1}(\gamma)$ for $\gamma \in H_{0}\left(\left(S^{1}, M^{n}\right)\right.$.

This lemma is an immediate consequence of the well-known inequality

$$
\int_{0}^{1} f g d x \leq \sqrt{\int_{0}^{1} f^{2} d x} \sqrt{\int_{0}^{1} g^{2} d x}
$$

where $f, g \in L_{2}[0,1]$.

Lemma 14. There exists a positive constant $T$ such that

$$
E_{1}(\gamma) \leq\left(T+\sqrt{T^{2}+4 \varepsilon S_{\varepsilon, \tau}}\right)^{2} / 4 \varepsilon^{2} .
$$

Proof of Lemma 14. Since the manifold $M^{n}$ is compact, there exists a constant $T$ such that

$$
\left|A_{j} v^{j}\right| \leq T|v|
$$

for any $x \in M^{n}, v \in T_{x} M^{n}$. 
Since the functional $E_{\tau}$ is non-negative,

$$
\varepsilon E_{1}+\Phi \leq \varepsilon E_{1}+E_{\tau}+\Phi=S_{\varepsilon, \tau}
$$

Now it is easy to see that

$$
\varepsilon E_{1}-T E_{0} \leq \varepsilon E_{1}-|\Phi| \leq S_{\varepsilon, \tau},
$$

and, by Lemma 13,

$$
\varepsilon E_{1}-T E_{0} \leq \varepsilon E_{1}-T \sqrt{E_{1}} \leq S_{\varepsilon, \tau} .
$$

Lemma 14 follows from the last inequality.

Lemma 15. Let $\varepsilon>0,0<\tau<1$. Then the functional

$$
S_{\varepsilon, \tau}: H_{0}\left(S^{1}, M^{n}\right) \rightarrow M^{n}
$$

satisfies the (PS) conditions.

Proof of Lemma 15. It is sufficient to show that for every given sequence $\left\{\overline{\gamma_{j}}\right\} \subset$ $H_{0}\left(S^{1}, M^{n}\right)$ such that

1) there exists constant $K_{0}$ such that $S_{\varepsilon, \tau}\left(\gamma_{j}\right)<K_{0}<+\infty$ for $j=1,2, \ldots$, and

2) $\left\|\delta S_{\varepsilon, \tau}\left(\overline{\gamma_{j}}\right)\right\| \rightarrow 0$ as $j \rightarrow+\infty$,

there exists a subsequence $\left\{\gamma_{j}\right\}$ which converges to a critical point of $S_{\varepsilon, \tau}$.

Here we denote by $\left\|\delta S_{\varepsilon, \tau}\right\|$ a norm of the variational differential:

$$
\left\|\delta S_{\varepsilon, \tau}(\gamma)\right\|=\sup \left\{\left|\delta S_{\varepsilon, \tau}(\nu)\right|: \nu \in T_{\gamma} H_{0}\left(S^{1}, M^{n}\right),\|\nu\|=1\right\}
$$

Let us consider such a sequence $\left\{\overline{\gamma_{j}}\right\}$.

By Lemma 14, there exists a constant $K_{1}$ such that $E_{1}\left(\overline{\gamma_{j}}\right) \leq K_{1}$ for $j=1,2, \ldots$

Let us denote by $d=d_{M}$ the distance function, on $M^{n}$, generated by the Riemannian metric $g_{i j}$. By the Cauchy-Schwarz inequality,

$$
d\left(\bar{\gamma}_{i}\left(t_{0}\right), \bar{\gamma}_{i}\left(t_{1}\right)\right) \leq \sqrt{2\left|t_{0}-t_{1}\right| E_{1}\left(\bar{\gamma}_{i}\right)} \leq \sqrt{2 K_{1}\left|t_{0}-t_{1}\right|} .
$$

The last inequality implies the sequence $\left\{\bar{\gamma}_{j}\right\}$ is equicontinuous and, since the sequence $\left\{\bar{\gamma}_{j}\left(t_{0}\right)\right\}$ is relatively compact for any $t_{0}$, then, by the Arzela-Ascoli theorem, there exists a subsequence $\left\{\gamma_{k}\right\} \subset\left\{\bar{\gamma}_{j}\right\}$ which converges to some contour $\gamma^{*} \in C^{0}\left(S^{1}, M^{n}\right)$ in the space $C^{0}\left(S^{1}, M^{n}\right)$.

It remains to prove that this $\left.\gamma^{*} \in H_{(} S^{1}, M^{n}\right)$ satisfies

$$
\left\|\delta S_{\varepsilon, \tau}\left(\gamma^{*}\right)\right\|=0
$$

Let us consider a small perturbation $\omega$ of the contour $\gamma^{*}$ such that

1) $\omega \in C^{\infty}\left(S^{1}, M^{n}\right)$;

2) $\gamma_{j}(t)=\exp \left(\omega(t), \xi_{j}(t)\right)$ where $\xi_{j} \in T_{\omega} H_{0}\left(S^{1}, M^{n}\right)$ and $\left|\xi_{j}(t)\right|_{M} \leq K_{2}$ for any $t \in[0,1], j \geq j_{0}$;

3) the exponential mapping $\exp : T_{\omega(t)} M^{n} \rightarrow M^{n}$ is a diffeomorphism onto its image on balls $|\eta|_{M} \leq 10 K_{2}<\operatorname{inj}\left(M^{n}\right)$ for any $t \in[0,1]$, and on every such ball the mappings

$$
\frac{\partial \exp (\omega(t), \eta)}{\partial x}: T_{\omega(t)} M^{n} \rightarrow T_{\exp (\omega(t), \eta)} M^{n}
$$

and

$$
\frac{\partial \exp (\omega(t), \eta)}{\partial v}: T_{\eta} T_{\omega(t)} M^{n} \rightarrow T_{\exp (\omega(t), \eta)} M^{n}
$$

are invertible. 
Without loss of generality, we assume that $j_{0}=1$.

Since $\gamma_{j} \rightarrow \gamma^{*}$ in $C^{0}\left(S^{1}, M^{n}\right), \xi_{j} \rightarrow \xi^{*}$ in the space of $C^{0}$-vector-fields:

$$
\rho_{j}=\max \left|\xi_{j}(t)-\xi^{*}(t)\right|_{M} \rightarrow 0
$$

as $j \rightarrow+\infty$, and we derive now that

$$
\int_{0}^{1}\left|\xi_{j}(t)-\xi^{*}(t)\right|^{2} d t \rightarrow 0
$$

as $j \rightarrow+\infty$.

It remains to prove that

$$
\int_{0}^{1}\left|\nabla_{\omega} \xi_{i}(t)-\nabla_{\omega} \xi_{j}(t)\right|^{2} d t \rightarrow 0
$$

as $i, j \rightarrow+\infty$, where $\nabla_{\omega}$ is a covariant derivation along the contour $\omega$.

For the sake of simplicity, let us put

$$
\begin{aligned}
A_{1, j}(t)=\frac{\partial \exp \left(\omega(t), \xi_{j}(t)\right)}{\partial x}, & A_{2, j}(t)=\frac{\partial \exp \left(\omega(t), \xi_{j}(t)\right)}{\partial v}, \\
A_{1}(t)=\frac{\partial \exp \left(\omega(t), \xi^{*}(t)\right)}{\partial x}, & A_{2}(t)=\frac{\partial \exp \left(\omega(t), \xi^{*}(t)\right)}{\partial v} .
\end{aligned}
$$

Let us represent $\dot{\gamma}_{j}(t)$ in the following form:

$$
\dot{\gamma}_{j}(t)=A_{1, j}(t) \dot{\omega}(t)+A_{2, j}(t) \nabla_{\omega} \xi_{j}(t) .
$$

It follows from this representation that

$$
\nabla_{\omega} \xi_{j}=A_{2, j}^{-1}\left(\dot{\gamma}_{j}-A_{1, j} \dot{\omega}\right)
$$

Since the right-hand side of this equality is square integrable (i.e. $\in L_{2}$ ) and since $S_{\varepsilon, \tau}\left(\gamma_{j}\right)$ are uniformly bounded $\left(<K_{0}\right)$, using Lemma 14 and the representation of $\gamma_{j}$ in the exponential form $\left(\gamma_{j}=\exp \left(\omega, \xi_{j}\right)\right)$, we conclude that

$$
\left\|\xi_{j}\right\|_{H}=\int_{0}^{1}\left(\left|\xi_{j}(t)\right|^{2}+\left|\nabla_{\omega} \xi_{j}(t)\right|^{2}\right) d t \leq K_{3}<+\infty
$$

where $K_{3}$ is some constant.

Let us introduce the vector-fields $u_{i j}$ along $\gamma_{i}$, defining them by the following formula:

$$
\gamma_{j}(t)=\exp \left(\gamma_{i}(t), u_{i j}(t)\right)
$$

We obtain that

$$
\nabla_{i} u_{i j}=\left(\frac{\partial \exp \left(\gamma_{i}, u_{i j}\right)}{\partial v}\right)^{-1}\left(A_{2, j} \nabla_{\omega} \xi_{j}-\frac{\partial \exp \left(\gamma_{i}, u_{i j}\right)}{\partial x} A_{2, i} \nabla_{\omega} \xi_{i}\right)+v_{i j}
$$

where

$$
v_{i j}=\left(\frac{\partial \exp \left(\gamma_{i}, u_{i j}\right)}{\partial v}\right)^{-1}\left(A_{1, j}-\frac{\partial \exp \left(\gamma_{i}, u_{i j}\right)}{\partial x} A_{1, i}\right) \dot{\omega}
$$

and $\nabla_{i}$ is the covariant derivation along $\gamma_{i}$.

Since by the construction the contours $\gamma_{i}$ lie in a sufficiently small neighborhood of $\omega$, we assume that the norms of the mappings

$$
\frac{\partial \exp \left(\gamma_{i}, u_{i j}\right)}{\partial x}, \quad \frac{\partial \exp \left(\gamma_{i}, u_{i j}\right)}{\partial v}
$$


and others which are inverse to them are bounded by some constant. Let us note also that

$$
v_{i j}=O\left(\rho_{i j}\right)=\max \left|\xi_{i}(t)-\xi_{j}(t)\right|,
$$

and, thus, there exists a constant $K_{4}$ such that

$$
\int_{0}^{1}\left(\left|u_{i j}(t)\right|^{2}+\left|\nabla_{i} u_{i j}(t)\right|^{2}\right) d t \leq K_{4}<+\infty
$$

Let us find an explicit formula for

$$
w_{i j}=\delta S_{\varepsilon, \tau}\left(\gamma_{i}\right)\left(u_{i j}\right)+\delta S_{\varepsilon, \tau}\left(\gamma_{j}\right)\left(u_{j i}\right) .
$$

By Lemmas 2 and 3,

$$
\begin{aligned}
\delta S_{\varepsilon, \tau}\left(\gamma_{i}\right)\left(u_{i j}\right)=\int_{0}^{1}\{ & \left(2 \varepsilon+(1+\tau)\left|A_{1, i} \dot{\omega}+A_{2, i} \nabla_{\omega} \xi_{i}\right|^{\tau-1}\right) \\
& \left.\times\left(A_{1, i} \dot{\omega}+A_{2, i} \nabla_{\omega} \xi_{i}, \nabla_{i} u_{i j}\right)+F_{k l} u_{i j}^{k} \dot{\gamma}_{i}^{l}\right\} d t .
\end{aligned}
$$

Since $\left|u_{i j}\right|=O\left(\rho_{i j}\right)$, we assume that

$$
\begin{aligned}
\delta S_{\varepsilon, \tau}\left(\gamma_{i}\right)\left(u_{i j}=\int_{0}^{1}\right. & \left(2 \varepsilon+(1+\tau)\left|A_{1} \dot{\omega}+A_{2} \nabla_{\omega} \xi_{i}\right|^{\tau-1}\right) \\
& \times\left(A_{1} \dot{\omega}+A_{2} \nabla_{\omega} \xi_{i}, A_{2}\left(\nabla_{\omega} \xi_{j}-\nabla_{\omega} \xi_{i}\right)\right) d t+O\left(\rho_{i j}\right) .
\end{aligned}
$$

Now let us prove the following auxiliary proposition.

Proposition 1. If $u, v \in R^{n} \backslash\{0\}$ and $0<\tau<1$, then

$$
\left(|u|^{2}-(u, v)\right)|u|^{\tau-1}+\left(|v|^{2}-(u, v)\right)|v|^{\tau-1} \geq 0 .
$$

Proof of Proposition 1. If $(u, v) \leq 0$ then the claim of proposition is evident. We are left with the case when $(u, v)=\beta|u||v|, 0 \leq \beta \leq 1$. Without loss of generality, we assume that $|u| /|v|=k \geq 1$. Let us divide the left-hand side of equality (3.5) by $|v|^{\tau+1}$ and reduce our problem to proving the inequality

$$
k^{1+\tau}-k^{\tau} \beta \geq k \beta-1 .
$$

But $k^{\tau}(k-\beta) \geq(k-\beta)$, and, thus, it is sufficiently to prove that $(k-\beta) \geq(k \beta-1)$. The last inequality is equivalent to the following:

$$
k(1-\beta) \geq(\beta-1),
$$

which holds since $k \geq 1$ and $0 \leq \beta \leq 1$.

Proposition 1 follows.

Let us apply inequality (3.5) to vectors $u(t)=A_{1}(t) \dot{\omega}(t)+A_{2}(t) \nabla_{\omega} \xi_{i}(t)$ and $v(t)=A_{1}(t) \dot{\omega}(t)+A_{2}(t) \nabla_{\omega} \xi_{j}(t)$ from $T_{\gamma^{*}(t)} M^{n}$. We obtain that

$$
D_{1, i j}=\int_{0}^{1}\left(|u|^{\tau+1}(u, v-u)+|v|^{\tau-1}(v, u-v)\right) d t \leq 0 .
$$

By (3.4),

$$
w_{i j}=D_{1, i j}+D_{2, i j}+O\left(\rho_{i j}\right)
$$

where

$$
\begin{aligned}
D_{2, i j}=\int_{0}^{1} 2 \varepsilon\{ & \left(A_{1} \dot{\omega}+A_{2} \nabla \omega \xi_{i}, A_{2} \nabla_{\omega}\left(\xi_{j}-\xi_{i}\right)\right. \\
& \left.+\left(A_{1} \dot{\omega}+A_{2} \nabla_{\omega} \xi_{j}, A_{2} \nabla_{\omega}\left(\xi_{i}-\xi_{j}\right)\right)\right\} d t .
\end{aligned}
$$


From the expression for $D_{2, i j}$ we conclude that

$$
-D_{2, i j}=2 \varepsilon \int_{0}^{1}\left|A_{2} \nabla_{\omega}\left(\xi_{i}-\xi_{j}\right)\right|^{2} d t .
$$

Now it follows from (3.4) that

$$
\begin{gathered}
2 \varepsilon \int_{0}^{1}\left|A_{2} \nabla_{\omega}\left(\xi_{i}-\xi_{j}\right)\right|^{2} d t \leq-w_{i j}+O\left(\rho_{i j}\right) \\
\leq R_{i j}=K_{4}\left(\left\|\delta S_{\varepsilon, \tau}\left(\gamma_{i}\right)\right\|+\left\|\delta S_{\varepsilon, \tau}\left(\gamma_{j}\right)\right\|\right)+O\left(\rho_{i j}\right) .
\end{gathered}
$$

But $R_{i j} \rightarrow 0$ as $i, j \rightarrow+\infty$, and, thus,

$$
\int_{0}^{1}\left|A_{2} \nabla_{\omega}\left(\xi_{i}-\xi_{j}\right)\right|^{2} d t \rightarrow 0
$$

as $i, j \rightarrow+\infty$, which in turn leads to

$$
\int_{0}^{1}\left|\nabla_{\omega} \xi_{i}-\nabla_{\omega} \xi_{j}\right|^{2} d t \rightarrow 0
$$

as $i, j \rightarrow+\infty$.

Using that and the fact that

$$
\int_{0}^{1}\left|\xi_{i}-\xi_{j}\right|^{2} d t \rightarrow 0, \quad i, j \rightarrow+\infty
$$

we deduce that the sequence $\left\{\xi_{i}\right\}$ is convergent in $T_{\omega} H_{0}\left(S^{1}, M^{n}\right)$ and, thus, converges to the vector-field $\xi^{*} \in T_{\omega} H_{0}\left(S^{1}, M^{n}\right)$ such that

$$
\gamma^{*}=\exp \left(\omega, \xi^{*}\right) \in H_{0}\left(S^{1}, M^{n}\right) \text { and }\left\|\delta S_{\varepsilon, \tau}\left(\gamma^{*}\right)\right\|=0,
$$

i.e. the contour $\gamma^{*}$ is the extremal of the functional $S_{\varepsilon, \tau}$.

Lemma 15 is proved.

C) Existence of critical points. First we recall the definition of the Ricci curvature tensor and introduce its analogue for fields defined by 2 -forms $F$.

Let $R_{j k l}^{i}$ be the Riemann curvature tensor and $\left\{e_{1}, \ldots, e_{n}\right\}$ an orthonormal basis, in the tangent space $T_{x} M^{n}$. Then the Ricci curvature tensor is defined by the formula

$$
K(u, v)=-\sum_{\alpha=1}^{n}\left(R\left(u, e_{\alpha}\right) v, e_{\alpha}\right)
$$

This definition does not depend on the choice of such a basis, because this tensor can be interpreted as the trace of the linear transformation

$$
\zeta \rightarrow R(u, \zeta) v \text {. }
$$

Let us also consider the tensor

$$
G(u, v, w)=\left(\nabla_{u} F\right)_{i j} v^{j} w^{k} .
$$

We can now obtain another tensor,

$$
\hat{G}(u, v, w)=(G(u, v, w)+G(v, u, w)) / 2,
$$

which is symmetric with respect to pair $(u, v)$ and satisfies

$$
\hat{G}(u, u, w)=G(u, u, w) .
$$


Now we introduce the tensor

$$
H(w)=\sum_{\alpha=1}^{n} \hat{G}\left(e_{\alpha}, e_{\alpha}, w\right)
$$

in the same manner which we used to introduce the Ricci curvature tensor. This definition is also independent of the choice of basis $\left\{e_{\alpha}\right\}$, because this tensor can be interpreted as the trace of the linear transformation

$$
\zeta^{i} \rightarrow g^{i k} \hat{G}_{j k l} \zeta^{j} w^{l},
$$

where

$$
\hat{G}_{j k l} u^{j} v^{k} w^{l}=\hat{G}(u, v, w) .
$$

Lemma 16. Let the function

$$
f(v)=K(v, v) /|v|-H(v)
$$

be positive on the subset of non-zero vectors, let $\gamma$ be a critical point of the functional $S_{\varepsilon, \tau}$ where $\tau<1$, and let $\mu(\gamma)$ be its Morse index. Then

$$
\text { length }(\gamma) \leq \max \left\{1, \frac{4 n\left((\mu(\gamma) \pi)^{2}\right.}{\Delta}\right\}
$$

where

$$
\Delta=\min _{v \in T M^{n},|v|=1}(K(v, v) /|v|-H(v)) .
$$

Proof of Lemma 16. Let $k>\mu(\gamma)$.

Let $\theta_{1}(t), \ldots, \theta_{n}(t)$ be a family of parallel orthonormal vector-fields along $\gamma$. Since in general $\theta_{i}(0) \neq \theta_{i}(1)$, let us define for every natural number $k$ the family $\beta_{j k}(t)$ by the following formulas:

$$
\beta_{j k}(t)=\sin (\pi k t) \theta_{j}(t)
$$

and

$$
\beta_{j k m}(t)= \begin{cases}\beta_{j k}(t), & \frac{m-1}{k} \leq t \leq \frac{m}{k}, \\ 0, & \text { otherwise. }\end{cases}
$$

These vector-fields lie in $H_{0}\left(S^{1}, M^{n}\right)$ (i.e. in $T_{\gamma} H_{0}\left(S^{1}, M^{n}\right)$ ).

By using elementary computations we obtain that

$$
\left(\beta_{j k m}, \beta_{j^{\prime} k^{\prime} m^{\prime}}\right)_{H}=\int_{0}^{1}\left(\left(\beta_{j k m}, \beta_{j^{\prime} k^{\prime} m^{\prime}}\right)+\left(\frac{D}{\partial t} \beta_{j k m}, \frac{D}{\partial t} \beta_{j^{\prime} k^{\prime} m^{\prime}}\right)\right) d t=0
$$

if the triples $(j, k, m)$ and $\left(j^{\prime}, k^{\prime}, m^{\prime}\right)$ do not coincide.

Since

we conclude that

$$
\frac{D}{\partial t} \beta_{j k}(t)=k \pi \cos (\pi k t) \theta_{j}
$$

$$
\begin{gathered}
\left(\frac{D}{\partial t}\left(\beta_{j k}(t), \frac{D}{\partial t} \beta_{j k}(t)\right)=(k \pi)^{2} \cos ^{2}(\pi k t),\right. \\
\left(\frac{D}{\partial t} \beta_{j k}(t), \dot{\gamma}(t)\right)=k \pi \cos (\pi k t)\left(\theta_{j}(t), \dot{\gamma}(t)\right), \\
F_{r s} \beta_{j k}^{r}\left(\frac{D}{\partial t} \beta_{j k}\right)^{s}=\sin \pi k t F_{r s} \beta_{j k}^{r}\left(\frac{D \theta_{j}}{\partial t}\right)^{s}+\pi k F_{r s} \beta_{j k}^{r} \beta_{j k}^{s} \equiv 0
\end{gathered}
$$


With the help of these formulas let us compute

$$
C_{k m}=\sum_{j=1}^{n} \delta^{2} S_{\varepsilon, \tau}(\gamma)\left(\beta_{j k m}, \beta_{j k m}\right) .
$$

For the sake of simplicity, let us denote by $L$ the length of the contour $\gamma$.

By Lemma 6,

$$
\begin{aligned}
C_{k m}= & 2 \varepsilon \int_{(m-1) / k}^{m / k}\left(n(k \pi)^{2} \cos ^{2}(\pi k t)-K(\dot{\gamma}, \dot{\gamma})\right) d t \\
& +(1+\tau) L^{\tau-1} \int_{(m-1) / k}^{m / k}\left((\tau-1)(k \pi)^{2} \cos (\pi k t)^{2}+n(k \pi)^{2} \cos ^{2}(\pi k t)\right. \\
& +\int_{(m-1) / k}^{m / k}\left(\sum_{j=1}^{n} \sin ^{2}(\pi k t)\left(\nabla_{i} F\right)_{r s} \theta_{j}^{i} \theta_{j}^{r} \dot{\gamma}^{s}\right) d t \\
= & \left.\frac{2 n \varepsilon(k \pi)^{2}+(1+\tau)(n-1+\tau)(k \pi)^{2} L^{\tau-1}}{2 k} \sin ^{2}(\pi k t)\right) d t \\
& -\left(2 \varepsilon+(1+\tau) L^{\tau-1}\right) \int_{(m-1) / k}^{m / k} \sin ^{2}(\pi k t) K(\dot{\gamma}, \dot{\gamma}) d t \\
& +\int_{(m-1) / k}^{m / k}\left(\sum_{j=1}^{n} \sin ^{2}(\pi k t)\left(\nabla_{i} F\right)_{r s} \theta_{j}^{i} \theta_{j}^{r} \dot{\gamma}^{s}\right) d t .
\end{aligned}
$$

Since $\varepsilon, \tau<1$, we conclude that if $L>1$ then

$$
\begin{aligned}
C_{k m} \leq & \frac{2 n(k \pi)^{2}}{k}-L^{-1} \int_{(m-1) / k}^{m / k} \sin ^{2}(\pi k t) K(\dot{\gamma}, \dot{\gamma}) d t \\
& +\int_{(m-1) / k}^{m / k} \sin ^{2}(\pi k t)\left(\sum_{j=1}^{n}\left(\nabla_{i} F\right)_{r s} \theta_{j}^{i} \theta_{j}^{r} \dot{\gamma}^{s}\right),
\end{aligned}
$$

i.e.

$$
\begin{aligned}
C_{k m} & \leq 2 n k \pi^{2}-L^{-1} \int_{(m-1) / k}^{m / k} \sin ^{2}(\pi k t) K(\dot{\gamma}, \dot{\gamma}) d t+\int_{(m-1) / k}^{m / k} \sin ^{2}(\pi k t) H(\dot{\gamma}) d t \\
& =2 n k \pi^{2}-\int_{(m-1) / k}^{m / k} \sin ^{2}(\pi k t)(K(\dot{\gamma}, \dot{\gamma}) /|\dot{\gamma}|-H(\dot{\gamma})) d t \\
& \leq 2 n k \pi^{2}-\frac{L \Delta}{2 k} .
\end{aligned}
$$

Thus, if

$$
L>\max \left\{1, \frac{4 n(k \pi)^{2}}{\Delta}\right\},
$$

then $C_{k m}<0$ for every $m=1, \ldots, k$.

Therefore for every $m$ there exists $j(m)$ such that

$$
\delta^{2} S_{\varepsilon, \tau}(\gamma)\left(v_{m}, v_{m}\right)<0
$$

where $v_{m}=\beta_{j(m) k m}$.

Now it is easy to see that

1) $\delta^{2} S_{\varepsilon, \tau}(\gamma)\left(v_{i}, v_{j}\right)=0$ for $i \neq j$; 
2) $\left(v_{i}, v_{j}\right)_{H}=\delta_{i j}$.

From the existence of such a family $\left\{v_{j}\right\}$ it immediately follows that the Morse index $\gamma$ is not less than $k$, which contradicts the definition of $\mu(\gamma)$.

Lemma 16 is proved.

Lemma 17. Let $\left\{\varepsilon_{k}, \tau_{k}\right\}$ be a sequence of pairs of positive sufficiently small numbers such that

$$
\lim _{k \rightarrow \infty}\left(\varepsilon_{k}, \tau_{k}\right)=(0,0)
$$

and $\left\{\overline{\gamma_{k}}\right\}$ be a sequence of points from $H_{0}\left(S^{1}, M^{n}\right)$ such that

1) $0<K_{0} \leq E_{1}\left(\overline{\gamma_{k}}\right) \leq K_{1}<+\infty$ where $K_{0}, K_{1}$ are some constants that do not depend on $k$;

2) $\delta S_{\varepsilon_{k}, \tau_{k}}\left(\overline{\gamma_{k}}\right)=0$.

Then there exists a subsequence $\left\{\gamma_{l}\right\} \subset\left\{\overline{\gamma_{k}}\right\}$ which converges in $H_{0}\left(S^{1}, M^{n}\right)$ to the critical point of the functional $S=\left(E_{0}+\Phi\right)$ :

$$
\begin{gathered}
\lim _{l \rightarrow \infty} \gamma_{l}=\gamma_{\infty}, \\
\delta\left(E_{0}+\Phi\right)\left(\gamma_{\infty}\right)=0 .
\end{gathered}
$$

Proof of Lemma 17. By Lemma 4, the contours $\overline{\gamma_{k}}$ are naturally parametrized, and, since the manifold $M^{n}$ is compact, we can pick a subsequence $\left\{\gamma_{l}\right\}$ such that, as $j \rightarrow \infty$,

1) the lengths of $\gamma_{l}$ converge to some constant $C>0$ :

$$
\text { length }\left(\gamma_{l}\right)=C_{l} \rightarrow C
$$

2) $\gamma_{l}(0)$ converges to some point $x_{0} \in M^{n}$;

3) $\dot{\gamma}_{l}$ converges to some vector $v \in T_{x_{0}} M^{n}$.

By Lemma 5, the motion of the point along every contour $\gamma_{l}$ is a periodic solution to the equation

$$
\frac{D}{\partial t} \dot{\gamma}_{l}^{j}=g^{j k} F_{k i} \dot{\gamma}_{l}^{i} /\left(2 \varepsilon_{l}+\left(1+\tau_{l}\right)\left|\dot{\gamma}_{l}\right|^{\tau_{l}-1}\right) .
$$

It follows from well-known theorems on the continuous dependence of solutions of differential equations on the initial data and coefficients that for every $t$ the sequence of points $\gamma_{l}(t)$ converges to a point $\gamma_{\infty}(t)$ where the curve $\gamma_{\infty}$ satisfies the equation

$$
\frac{D}{\partial t} \dot{\gamma}_{\infty}^{j}=C g^{j k} F_{k i} \dot{\gamma}_{\infty}^{i}
$$

with the initial data

$$
\gamma_{\infty}(0)=x_{0}, \dot{\gamma}_{\infty}(0)=v
$$

Since the curves $\gamma_{l}$ are closed, we may conclude that the curve $\gamma_{\infty}$ is also closed; and since it satisfies equation (3.6), this closed curve is a critical point of the functional $S=\left(E_{0}+\Phi\right)$.

Lemma 17 is proved.

Theorem 1. Let there exists a contour $\gamma \in H_{0}\left(S^{1}, M^{n}\right)$ such that

$$
S(\gamma)=\int_{\gamma}\left(\sqrt{g_{i j} \dot{\gamma}^{i} \dot{\gamma}^{j}}+A_{i} \dot{\gamma}^{i}\right) d t<0 .
$$


Let also

$$
f(v)=K(v, v) /|v|-H(v)>0
$$

for $v \neq 0$. Then for every cycle $\alpha \in H_{l}\left(M^{n}\right) \backslash\{0\}$ there exists a sequence $\left\{\gamma_{\varepsilon_{k}, \tau_{k}}\right\}$ of critical points of the functional $S_{\varepsilon_{k}, \tau_{k}}$ such that

1) $\varepsilon_{k}, \tau_{k} \rightarrow 0$ as $k \rightarrow \infty$;

2) $S\left(\gamma_{\varepsilon_{k}, \tau_{k}}\right)=c_{\varepsilon, k}, \tau_{k}(\alpha)$;

3) index $\gamma_{\varepsilon_{k}, \tau_{k}} \leq(l+1)$;

4) the sequence $\gamma_{\varepsilon_{k}, \tau_{k}}$ converges to a non-point extremal of the functional $S$ as $k \rightarrow \infty$.

Proof of Theorem 1. By Lemmas 9 and 10,

$$
\operatorname{dim} H_{l+1}\left(H_{0}\left(S^{1}, M^{n}\right),\left\{S_{\varepsilon, \tau} \leq 0\right\} ; \mathbf{R}\right) \neq 0
$$

for sufficiently small values of $\varepsilon, \tau$. By Lemma 15 , the functionals $S_{\varepsilon, \tau}$ satisfy the Palais-Smale conditions, and, therefore, there exists a critical point $\gamma_{\varepsilon, \tau}$ such that its Morse index is less than or equal to $(l+1)$ and $S_{\varepsilon, \tau}\left(\gamma_{\varepsilon, \tau}\right)=c_{\varepsilon, \tau}(\alpha)$ (the critical values $c_{\varepsilon, \tau}$ were defined at Lemma 9$)$.

By Lemma 12, there exists a positive constant $K_{0}$ such that

$$
E_{1}\left(\gamma_{\varepsilon, \tau}\right) \geq K_{0}
$$

and, by Lemma 16, there exists a positive constant $K_{1}$ such that

$$
E_{1}\left(\gamma_{\varepsilon, \tau}\right)=E_{0}^{2}\left(\gamma_{\varepsilon, \tau}\right) \geq K_{1}
$$

Here we ought to take into account that all contours $\gamma_{\varepsilon, \tau}$ are naturally parametrized (see Lemma 4).

Now it immediately follows from Lemma 17 that there exists a sequence of $\left\{\gamma_{\varepsilon_{k}, \tau_{k}}\right\}$ which converges in $H_{0}\left(S^{1}, M^{n}\right)$ to a critical point $\gamma_{\infty}$ of the functional $S=\left(E_{0}+\Phi\right)$ as $k \rightarrow \infty$, and $E_{1}\left(\gamma_{\infty}\right)>K_{0}>0$ (i.e. the extremal $\gamma_{\infty}$ is not one-point).

Theorem 1 is proved.

Let us notice that the problem of distinguishing between critical points which we have obtained can be solved under additional conditions involving the Morse type of these critical points.

\section{D) On applications of Theorem 1.}

Definition. Let $\left(M_{1}, g^{(1)}, F^{(1)}\right), \ldots,\left(M_{k}, g^{(k)}, F^{(k)}\right)$ be Riemannian manifolds endowed with Riemannian metrics $g^{(i)}$ and 2 -forms $F^{(j)}$ respectively. We call the direct product of the Lagrangian systems $\left(M_{1}, g^{(1)}, F^{(1)}\right), \ldots,\left(M_{k}, g^{(k)}, F^{(k)}\right)$ the Lagrangian system of the same type defined on the direct product of these manifolds

$$
M^{*}=M_{1} \times \cdots \times M_{k}
$$

with the product metric and the 2 -form $F^{*}$ defined by the formula

$$
F^{*}=\sum_{j} p_{j}^{*}\left(F^{(j)}\right)
$$

where $p_{j}: M^{*} \rightarrow M_{j}$ are natural projections on the factors. 
Lemma 18. Let $M^{n}$ be a Riemannian manifold endowed with an exact non-zero 2-form $F$ such that inequality (3.7) holds. Then there exists an integer $k$ such that

1) the product of $k$ examples of the Lagrangian systems $\left(M^{n}, g_{i j}, F\right)$ satisfies (3.7);

2) on $\left(M^{n}\right)^{k}$, there exist closed smooth contours at which the functional

$$
S(\gamma)=\int_{\gamma}\left(|\dot{\gamma}|+A_{i}^{*} \dot{\gamma}^{i}\right) d t
$$

(where $d A^{*}=F^{*}$ ) takes negative values, i.e. this system satisfies the conditions of Theorem 1.

Proof of Lemma 18. Since the form $F$ is non-zero, there exists a loop $\gamma$ on $M^{n}$ such that

$$
\int_{\gamma} A_{i} \dot{\gamma}^{i} d t=R<0
$$

Let $k>(\text { length }(\gamma) / R)^{2}$. We consider a contour $\gamma^{*}$ on $\left(M^{n}\right)^{k}$ which projects onto the contour $\gamma$ under the projection onto every factor. It is easy to see that

$$
\text { length }\left(\gamma^{*}\right)=\sqrt{k} \text { length }(\gamma) \text {, }
$$

and

$$
\int_{\gamma^{*}} A_{i}^{*} \dot{\gamma}^{* i} d t=k \int_{\gamma} A_{i} \dot{\gamma}^{i} d t
$$

We then have

$$
S\left(\gamma^{*}\right)=\sqrt{k} \operatorname{length}(\gamma)-k \int_{\gamma} A_{i} \dot{\gamma}^{i} d t<0 .
$$

Lemma 18 is proved.

\section{E) On the existence of extremals of the functional $\mathrm{S}$.}

Theorem 2. Let $M^{n}$ be a closed Riemannian manifold endowed with a Riemannian metric $g_{i j}$ and an exact 2-form $F$ which satisfy the following condition:

$$
\min _{|v|=1}\{f(v)=K(v, v) /|v|-H(v)\}>0 .
$$

Then there exists a closed nontrivial extremal of the functional

$$
S(\gamma)=\int_{\gamma}\left(\sqrt{g_{i j} \dot{x}^{i} \dot{x}^{j}}+A_{i} \dot{x}^{i}\right) d t
$$

where $d A=F$.

Proof of Theorem 2. The real homologies of the pair $\left(H_{0}\left(S^{1}, M^{n}\right), M^{n}\right)$, where $M^{n} \subset H_{0}\left(S^{1}, M^{n}\right)$, are nontrivial ([K1]). Let us pick some nontrivial cycle $u \in$ $H_{l}\left(H_{0}\left(S^{1}, M^{n}\right), M^{n} ; \mathbf{R}\right)$.

It is evident that there exists a sequence of pairs of positive numbers $\left\{\varepsilon_{k}, \tau_{k}\right\}$ such that

1) $\varepsilon_{k}, \tau_{k} \rightarrow 0$ as $k \rightarrow \infty$;

2) the functionals $S_{\varepsilon_{k}, \tau_{k}}$ are positive outside of the set of one-point contours, or for every one of them there exists a non-one-point contour $\gamma_{k} \in H_{0}\left(S^{1}, M^{n}\right)$ such that $S_{\varepsilon_{k}, \tau_{k}}\left(\gamma_{k}\right)=0$. 
These functionals $S_{\varepsilon_{k}, \tau_{k}}$ satisfy the PS conditions (see Lemma 15). Thus, if the first case holds for every one of them, there exists a non-one-point extremal with a Morse index less than or equal to $l$ (this extremal corresponds, in the usual manner, to the cycle $u$ ); otherwise for every one of them there exists a non-one-point extremal with Morse index equal to 1 (by Lemma 9).

We can then repeat the arguments of the proof of Theorem 1 and derive Theorem 2 .

Theorem 2 is proved.

The second author (I.A.T.) was supported by the International Science Foundation (ISF) (grant no. M1E300).

\section{REFERENCES}

[A] Arnold V.I., First steps in symplectic topology, Russian Mathematical Surveys 41 : 6 (1986), 1-21. MR 89d:58034

[B] Bahri A., Pseudo-orbits of contact forms, Pitman Research Notes in Mathematics Series. V. 173. Longman Scientific and Technical, 1988. MR 90i:58160

[CZ] Conley C., Zehnder E., The Birkhoff-Lewis fixed point theorem and a conjecture of V.I. Arnold, Invent. Math. 73 (1983), 33-49. MR 85e:58044

[G1] Ginzburg V.L., New generalizations of the geometrical theorem of Poincaré, Funct. Anal. Appl. 21 (1987), 100-106. MR 89f:58057

[G2] Ginzburg V.L., On the existence and non-existence of closed trajectories for some hamiltonian flows, Math. Zeitschrift. 223 (1996), 397-409. MR 97i:58144

[GN] Grinevich P.G., Novikov S.P., Nonselfintersecting magnetic orbits on the plane. Proof of the overthrowing of cycles principle, Topics in Topology and Mathematical Physics, AMS Translations - Series 2. Vol. 170, 1995, pp. 59-82. MR 96j:58031

[H] Hedlund G.A., Fuchsian groups and transitive horocycles, Duke Math. J. 2 (1936), 530542 .

[K1] Klingenberg, W., Lectures on closed geodesics, Grundlehren Math. Wiss., Bd. 230, Springer, Berlin and New York, 1978. MR 57:17563

[K2] Klingenberg, W., Riemannian geometry, 2nd ed., Studies in Math., de Gruter, Berlin and New York, 1995. MR 95m:58003

[Ko] Kozlov V.V., Calculus of variations in the large and classical mechanics, Russian Mathematical Surveys 40 : 2 (1985), 37-71. MR 86m:58053

[M] Milnor, J., Morse theory, Ann. Math. Studies, no. 51, Princeton Univ. Press, Princeton, N.J., 1963. MR 29:634

[N1] Novikov, S.P., Multivalued functions and functionals. An analogue of Morse theory, Soviet Math. Dokl. 24 (1981), 222-226. MR 83a:58025

[N2] Novikov, S.P., Variational methods and periodic solutions of equations of Kirchhoff type. II, Functional Anal. Appl. 15 (1981), 263-274. MR 83a:58026b

[N3] Novikov, S.P., Hamiltonian formalism and a multivalued analogue of Morse theory, Russian Math. Surveys 37 : 5 (1982), 1-56. MR 84h:58032

[NS] Novikov, S.P., Shmel'tser, I., Periodic solutions of the Kirchhoff equations for the free motion of a rigid body in a liquid, and the extended Lyusternik-Schnirelmann-Morse (LSM) theory.I, Functional Anal. Appl. 15 (1981), 197-207. MR 83a:58026a

[NT] Novikov, S.P., Taimanov I.A., Periodic extremals of multivalued or not everywhere positive functionals, Soviet Math. Dokl. 29 (1984), 18-20. MR 85i:58032

[PS] Palais, R., Smale, S., A generalized Morse theory, Bull. Amer. Math. Soc. 70 (1964), 165-171. MR 28:1634

[SU] Sacks J., Uhlenbeck K., The existence of minimal immersions of two-spheres, Ann. of Math. 113 (1981), 1-24. MR 82f:58035

[T1] Taimanov, I.A., The principle of throwing out cycles in Morse-Novikov theory, Soviet Math. Dokl. 27 (1983), 43-46. MR 84h:58038

[T2] Taimanov, I.A., Non-selfintersecting closed extremals of multivalued or not everywhere positive functionals, Math. USSR Izvestiya 38 (1992), 359-374. MR 92i:58036 
[T3] Taimanov, I.A., Closed non-selfintersecting extremals of multivalued functionals, Siberian Math. Journal 33 (1992), 686-692. MR 93j:49004

[T4] Taimanov, I.A., Closed extremals on two-dimensional manifolds, Russian Math. Surveys 47 : 2 (1992), 163-211. MR 93k:58050

Department of Mathematics, Rutgers University, New Brunswick, New Jersey 08903

Institute of Mathematics, 630090 Novosibirsk, Russia

E-mail address: taimanov@math.nsc.ru 